\title{
La voie du gamelan. Entretien avec Ki Mantle Hood
}

\section{Giovanni Giuriati et Ki Mantle Hood}

Traducteur : Ramèche Goharian

\section{OpenEdition}

\section{Journals}

Édition électronique

URL : http://journals.openedition.org/ethnomusicologie/1213

ISSN : 2235-7688

\section{Éditeur}

ADEM - Ateliers d'ethnomusicologie

Édition imprimée

Date de publication : 31 décembre 1995

Pagination : 193-214

ISBN : 2-8257-0537-3

ISSN : 1662-372X

\section{Référence électronique}

Giovanni Giuriati et Ki Mantle Hood, «La voie du gamelan. Entretien avec Ki Mantle Hood », Cahiers d'ethnomusicologie [En ligne], 8 | 1995, mis en ligne le 04 janvier 2012, consulté le 01 mai 2019. URL

http://journals.openedition.org/ethnomusicologie/1213

Ce document a été généré automatiquement le 1 mai 2019.

Tous droits réservés 


\title{
La voie du gamelan ${ }^{1}$. Entretien avec Ki Mantle Hood
}

\author{
Giovanni Giuriati et Ki Mantle Hood
}

Traduction : Ramèche Goharian

1 Ki Mantle Hood est une des figures marquantes de l'ethnomusicologie. L'Institut d'ethnomusicologie qu'il a fondé en 1961 à l'Université de Californie à Los Angeles (UCLA) et où il a enseigné jusqu'en 1974 a été longtemps considéré comme un modèle et une référence. Les idées de ce savant influencent depuis de nombreuses années les études ethnomusicologiques aux Etats-Unis et dans le reste du monde.

2 Hood est né le 24 juin 1918, à Springfield en Illinois. Après avoir joué du saxophone dans des orchestres de jazz et étudié la composition musicale, il s'est intéressé à la musique javanaise. Une bourse Fulbright lui permit de se rendre en Europe pour étudier avec Jaap Kunst. En 1954, il obtint son doctorat cum laude à l'Université d'Amsterdam avec une thèse sur la pratique modale javanaise intitulée The Nuclear Theme as a Determinant of Patet in Javanese Music. La même année, il fut nommé maître-assistant à UCLA. De 1956 à 1958, il fit des recherches en Indonésie où, grâce à une bourse Ford, il put étudier sur le terrain les cultures musicales de Java Central et de Bali. De retour à UCLA, il élabora le premier programme d'étude systématique en ethnomusicologie. L'Institut d'ethnomusicologie de UCLA est devenu, grâce à lui, un centre dynamique pour la toute nouvelle discipline qui faisait son entrée dans le monde académique. Toute une génération d'ethnomusicologues, qui enseignent aujourd'hui dans les grandes universités américaines, y ont fait leurs études. Parmi les nombreux professeurs qui ont enseigné à différents moments à l'Institut, on peut citer: Charles Seeger, Klaus Wachsmann, Colin McPhee, J. Kwabena Nketia, Ravi Shankar.

3 Premier centre d'enseignement pratique du gamelan javanais et balinais, l'Institut a donné naissance à une activité devenue aujourd'hui très répandue en Occident : on trouve en effet de nombreux groupes jouant du gamelan dans les départements d'ethnomusicologie d'Europe et d'ailleurs. C'est surtout sur l'enseignement pratique des musiques non-occidentales que l'on mettait l'accent à UCLA. Cette recherche de la «bi- 
musicalité ", de l'apprentissage pratique de la musique que l'on étudie, a été une des contributions majeures de Mantle Hood aux études ethnomusicologiques. Le fait de considérer la musique (en relation avec son contexte culturel) comme le principal sujet d'étude en ethnomusicologie constitue un autre apport méthodologique important de ce savant.

Passionné de musique javanaise, Hood a étudié les différents aspects de cette culture musicale. Ses recherches sur les systèmes d'accordage, l'improvisation, le système modal et l'histoire de la musique javanaise sont d'une importance capitale pour les études ethnomusicologiques et forment la base de la recherche sur les cultures musicales du Sud-Est asiatique.

Il s'est aussi beaucoup intéressé à l'étude du son. Depuis sa collaboration avec Charles Seeger à UCLA, dans les années soixante, il a participé au développement des différents modèles du Mélograph de Seeger. Son intérêt constant pour la recherche sur le son, suscité par la complexité sonore des musiques javanaises et balinaises, l'a poussé à proposer récemment une nouvelle perspective d'étude: la théorie quantique de la musique.

6 Hood vient d'être nommé professeur honoraire à la Faculté des arts créatifs de l'Université de West Virginia pour l'année académique 1995-1996. Dans sa belle villa d'Ellicott City près de Baltimore, il continue de communiquer, au milieu de ses gamelans de Java et de Bali, son intarissable énergie et un enthousiame contagieux à tous ses visiteurs. Actuellement il projette de créer un club privé de danse et de musique balinaises Seka Genta Semara pour jouer, sur le beau gamelan Semar Pegulingan, les compositions musicales de I Wayan Lotring, un des plus célèbres musiciens de Bali.

G.G.

Vous avez découvert assez tard l'ethnomusicologie, comme c'est souvent le cas dans notre domaine. Quelle était votre formation et comment et pourquoi vous êtes vous engagé sur la voie de l'ethnomusicologie?

Pour répondre complètement à votre question, je devrais vous raconter presque le tiers de ma vie ; je ne vous en indiquerai donc que quelques points principaux. J'ai fait de la musique dès mon enfance, dans l'Illinois. D'abord en chantant, puis comme clarinettiste et enfin dans le jazz, en jouant du saxophone ténor. Je suis devenu un " pro » à quinze ans et j'ai joué dans des boîtes de nuit, des hôtels et parfois dans de grands clubs situés à l'extérieur de la ville, que l'on appelait à l'époque des «relais». Il s'agissait d'orchestres syndiqués qui travaillaient en faisant alterner les interventions musicales avec des pauses de même durée. Il y avait généralement une loge pour les musiciens où je trouvais assez d'espace et de temps pour faire mes devoirs d'école. A la maison, je dormais par " quart ", c'est-à-dire de quatre heures du matin à sept heures où j'allais à l'école; de quatre heures de l'après-midi à huit heures, je dînais à la maison et je repartais pour la boîte de nuit. L'âge moyen des autres musiciens était de trente-cinq ans environ, ce qui fait qu'à quinze ans j'ai reçu un enseignement de jazz donné par des musiciens qui s'étaient déjà "retirés" d'orchestres connus : c'était une éducation merveilleusement concentrée. J'ai continué dans des orchestres de bal, en Californie (à une certaine époque, j'ai même été le chanteur de charme favori de Shirley Temple).

Un soir, un ami m'a appelé à Santa Monica, en Californie, pour me dire : "Annule tous tes projets pour ce soir! Il y a deux copains qui sont revenus du Sud-Est asiatique, tu dois absolument les rencontrer. » Nous avons passé la soirée à admirer une superbe exposition d'art asiatique où figuraient des enregistrements de gamelan de Java et de 
Bali. Cela, c'était le début. Quelques années plus tard, en guise d'introduction à un cours d'histoire de la musique, un professeur de UCLA a commencé par nous faire écouter de vieux disques 78 tours de Musique de l'Orient; ce fut ma deuxième découverte. Après une maîtrise en composition musicale (en ces temps-là il n'y avait pas de doctorat en composition), je suis allé en Hollande pour étudier avec Jaap Kunst. Comme compositeur, je percevais une logique musicale impressionnante dans les sons de Java et de Bali, mais je ne pouvais pas la comprendre. J'ai commencé en 1952 des recherches avec Kunst sur la pratique modale javanaise. Mais c'est seulement en 1966 que j'ai finalement compris les bases de l'improvisation de groupe. Après avoir obtenu mon doctorat en 1954, j'ai commencé mon premier travail de terrain à Bali et à Java en janvier 1957. J'avais pris avec moi des piles de papier à musique avec l'intention de composer régulièrement. Pendant ces deux années, je n'ai pas touché à ces papiers. Dix ans plus tard, je me suis remis à composer, cette fois avec beaucoup plus de facilité.

McPhee raconte une histoire semblable dans son livre $A$ House in Bali. Il est, lui aussi, parti comme compositeur à Bali, dans les années trente, mais il a très peu écrit pendant son séjour dans cette île. Etait-ce la fascination pour la complexité de cette musique qui, comme vous-même, l'a empêché de composer ou était-ce quelque chose d'autre?

Comme vous le savez, nous étions de très bons amis, Colin et moi, de 1958 environ jusqu'à sa mort en 1963. Inutile de dire que nous avons certainement passé des centaines d'heures à parler ensemble de gamelan. Votre question est intéressante parce que je pense que c'était un sujet que nous abordions avec réticence; nous comprenions parfaitement tous deux la cause de la longue période de sécheresse qui tarissait notre créativité. Nous étions tout simplement subjugués par la richesse des ressources de la musique de gamelan. Ce n'est pas vraiment que nous ayons été intimidés, mais nous nous trouvions devant un défi démesuré. Tant que je n'avais pas compris à fond, comme compositeur, les bases de la pratique du gamelan, je n'étais tout simplement pas en mesure de continuer à composer. Je crois que McPhee ressentait la même chose. Rappelez-vous qu'une de ses premières compositions après Bali, qui a d'ailleurs remporté un grand succès, a été « Tabuh-tabuhan ».

Vous êtes venu en Europe au début des années cinquante pour étudier l'ethnomusicologie. Pourquoi? Quel souvenir gardez-vous de Jaap Kunst comme savant et comme professeur?

Je suis d'abord allé chez Kunst avant de me rendre en Indonésie, parce que j'avais l'impression qu'il fallait que j'apprenne le plus possible de l'homme qui en savait le plus avant de passer du temps sur le terrain. C'était certainement la bonne décision à prendre.

En tant que professeur, savant et bientôt ami, Jaap Kunst a véritablement été un grand modèle. Dans la tradition des professeurs européens, Kunst était très différent de mes professeurs américains : il m'a laissé entièrement libre de trouver mon sujet et ma méthode de travail et m'a permis de développer mon esprit d'initiative. Dans la tradition de l'éducation hollandaise on ne mâchait certainement pas la tâche à l'étudiant. Une fois que j'ai eu compris cela, il m'a prodigué son temps, son énergie et toutes ses ressources. Sur le plan académique, Kunst avait eu son diplôme en droit. Au début je croyais que son approche de la recherche découlait de cette formation. Et je suis sûr que dans une certaine mesure c'était le cas. Mais peu à peu j'ai découvert que tous les savants hollandais, dont j'ai connu et admiré l'œuvre consacrée à l'Indonésie, avaient une approche analogue quelle qu'ait été leur discipline de base : je veux dire 
qu'ils étudiaient leur sujet sous tous les angles imaginables de sorte que, comme le disait Kunst, "si vous arrivez à prévoir toutes les questions et tous les défis, vous désarmerez tous vos critiques potentiels». C'est la conviction typique de tous les grands savants hollandais: j'ai tâché de la reproduire et de l'utiliser dans mon enseignement.

Vous venez de mentionner une «tradition des professeurs européens». Quelle est la différence, s'il y en a une, avec la méthode américaine de l'enseignement?

Fig. 1 : En apprenant un pathetan (prélude modal) sur un gender javanais, Solo, Java, 1957.

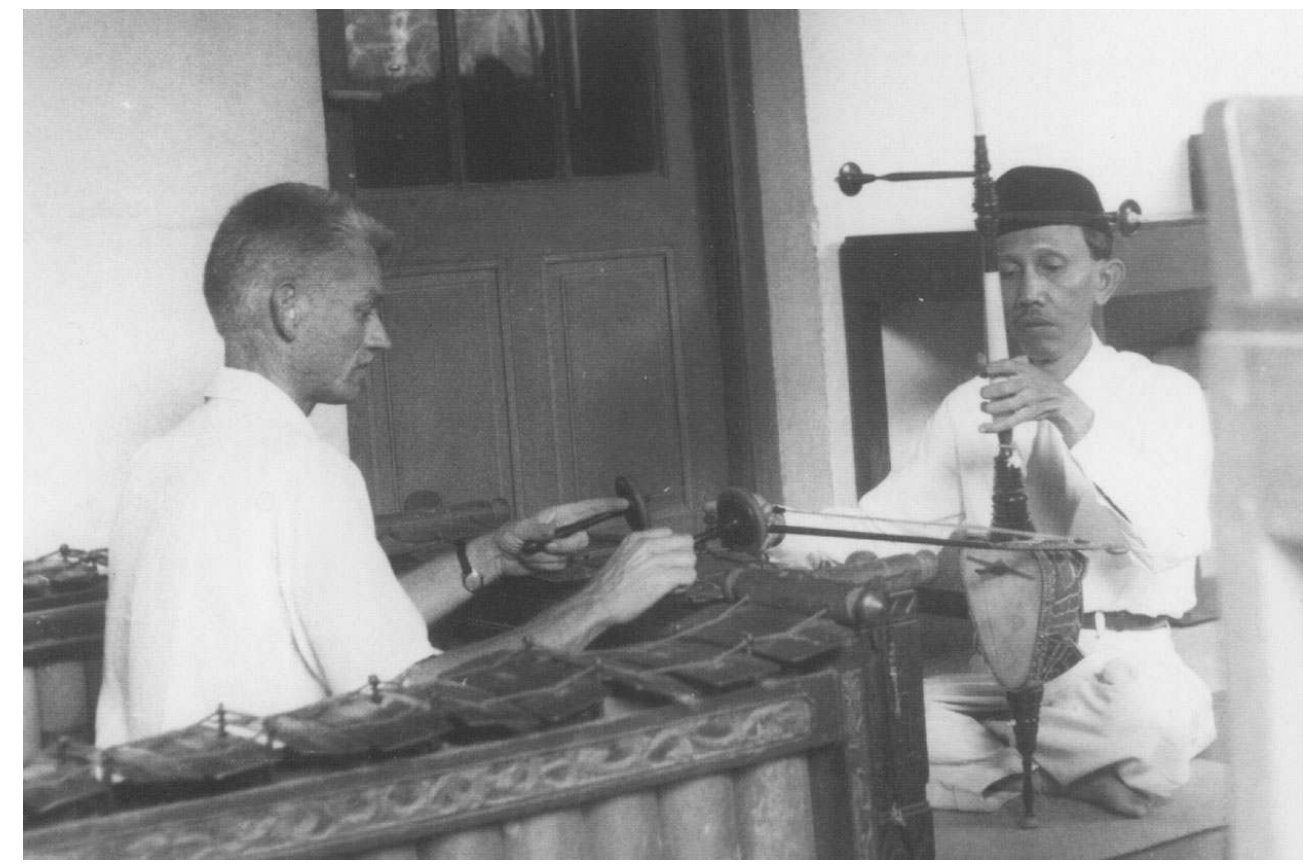

Je suis content que vous releviez la chose. En fait je parlais de l'enseignement et de la spécialisation en Hollande car j'en ai moi-même une expérience personnelle. Mais il me semble qu'il y a une attitude dans l'enseignement (pas nécessairement dans la recherche, comme j'ai pu l'observer chez les autres savants européens) que l'on pourrait très bien qualifier de «tradition européenne de l'enseignement ». Elle est vraiment différente de la tradition qui a cours aux Etats-Unis.

En Amérique, le «mentor » va si loin dans sa manière de guider l'étudiant qu'on peut dire qu'il lui mâche véritablement le travail. J'ai très vite compris qu'il était important de laisser l'étudiant avancé développer lui-même son esprit d'initiative et d'innovation dans sa recherche. Nous avons une expression américaine qui dit « coule ou nage » dont l'origine remonte à cette époque où l'on jetait simplement l'enfant à l'eau en lui laissant le choix entre couler ou apprendre tout de suite à nager. J'ai appris que cette attitude du professeur à l'égard de son étudiant était à la longue la plus bénéfique. Si on ne développe pas l'indépendance d'esprit et l'originalité dans la méthodologie pendant cette période cruciale de la recherche et de la rédaction d'une thèse, elles pourraient bien ne jamais plus se manifester. Aucun directeur de recherche, si bien intentionné soit-il, ne pourra jamais remplacer dans sa direction le merveilleux défi du « coule ou nage ». Je crois que tous mes doctorants peuvent témoigner qu'ils ont fait cette expérience et j'espère qu'ils appliqueront la même recette pour former leurs propres étudiants. Et cela n'est pas typiquement la « méthode américaine de l'enseignement ». 
A part Kunst, quelles sont les personnes que vous considérez comme vos maîtres?

J'aimerais citer trois personnalités qui m'ont marqué au temps où j'étais à l'école secondaire. La première, Suzanne Wilcox, enseignait la littérature anglaise (une aile de la bibliothèque et une rue de Springfield, en Illinois, portent aujourd'hui son nom). Elle était appréciée, en Angleterre, pour ses conférences sur Shakespeare. Elle a eu une grande influence sur ma façon d'écrire et de penser, depuis mon premier cours avec elle à l'âge de treize ans. La deuxième, Mademoiselle Graham, était écrivain et journaliste. Les journaux de Chicago ont souvent essayé de l'attirer dans cette ville. C'est elle qui m'a appris à regarder «le monde extérieur », au delà de mon Midwest natal. La troisième, dont j'ai malheureusement oublié le nom, était une historienne raffinée et élégante. Elle m'a fait comprendre la nécessité d'une perspective mondiale.

Comme jeune adulte, j'ai fortement subi l'influence de trois autres professeurs (quelle chance d'en avoir connu autant!). Les cinq années de cours privés que m'a donné le compositeur Ernst Toch ont non seulement été cruciales pour mon éducation musicale, mais m'ont aussi permis de me faire un ami intime. Il était docteur en philosophie et avait une rare sensibilité esthétique. Mon deuxième grand maître a naturellement été Jaap Kunst. Je crois que c'est principalement lui qui a formé mes méthodes de recherche et ma philosophie de l'enseignement. Le troisième a été Charles Seeger, le grand philosophe de la théorie de la parole et de la musique, qui est resté pendant onze ans, de l'âge de 75 à 86 ans, à l'Institut d'ethnomusicologie de UCLA. Grâce à lui j'ai continué à beaucoup apprendre sur les valeurs, sujet que l'on n'abordait pas souvent dans l'éducation supérieure. Ces trois savants sont devenus mes meilleurs amis.

Charles Seeger est une personnalité dont les idées et l'œuvre ne sont pas aussi connues qu'elles le mériteraient en Europe. Pourriez-vous le décrire brièvement, comme professeur et collègue.

Avec un immense plaisir ! Lorsque j'ai invité Charles à nous rejoindre à l'Institut, je lui ai dit qu'il n'aurait aucune obligation rédactionnelle ou autre, aucune échéance ou limitation à ce qu'il décidait d'entreprendre. Je lui ai simplement demandé d'assister aux séminaires et aux classes qu'il trouvait intéressants, de parler avec les étudiants quand il le voulait, bref d'être présent autant qu'il le désirait. Quel apport enrichissant pour nos étudiants avancés que la participation de ce grand savant, pendant ces onze années!

Je pense que ses capacités intellectuelles vraiment exceptionnelles étaient uniques à UCLA, et ce pour une raison particulière. Charles faisait partie de cette génération de savants en voie de disparition, formés à l'école de la philosophie grecque et latine et dont la curiosité intellectuelle les poussait dans tous les domaines imaginables. Je pense à la génération des George Bernard Shaw, G.K. Chesterton, Ford Madox Ford, W.H. Hudson, Charles Ran Kennedy, Forsythe. C'était un extraordinaire homme de science. En fait, il faudrait relire plusieurs fois chacun de ses écrits pour apprécier la finesse de son style et la précision de son raisonnement théorique. Il a consacré l'essentiel de sa réflexion au rapport qui existe entre la parole et la musique; il a montré qu'entre ces deux modes de communication il existe trois ordres de références: une même signification dans la parole et dans la musique, une signification similaire dans la parole et dans la musique, une signification différente dans la parole et dans la musique. Il prétendait - et certains d'entre nous sont d'accord avec lui - que les musicologues ont trop souvent tendance à faire des discours dans leur argumentation scientifique, sans 
se rendre compte qu'ils glissent imperceptiblement de l'un à l'autre de ces trois ordres, dans leurs écrits ou leurs raisonnements.

En tant que professeur et collègue, il participait régulièrement au fameux « Séminaire principal du mercredi » de l'Institut, où de quatre à sept professeurs de différentes disciplines débattaient avec une trentaine d'étudiants avancés des problèmes relatifs au monde de la musique. Il m'est presqu'impossible, dans un entretien aussi court, de rendre un juste hommage à une personnalité aussi remarquable que Charles Seeger.

Le programme d'ethnomusicologie que vous avez établi à UCLA est considéré comme un modèle du genre dans ce domaine par la richesse de ses approches et par la présence de quelques-unes des personnalités les plus éminentes de cette discipline. Pourriez-vous nous parler des débuts de ce projet.

Lorsque j'ai donné mon premier séminaire d'ethnomusicologie, au printemps 1955, je me suis trouvé face à des étudiants avancés, dans un domaine qui disposait de peu de sources, peu de livres appropriés dans l'immense bibliothèque de UCLA et peu de connaissances sur le monde de la musique en dehors de l'Europe et des Etats-Unis. Je me suis demandé ce que je pourrais faire pour aider ces jeunes gens à choisir un sujet original, à entreprendre de l'étudier et à leur faire sentir qu'ils s'engageaient dans une recherche extraordinaire.

Mais d'abord je dois encore dire ceci : Un ami entomologiste a mené, il y a longtemps, une expérience dans ce qu'il appelait l'« énergie négative ». Je ne vais pas essayer de vous expliquer sa thèse mais seulement mentionner la conclusion de son étude, où il avait obtenu des résultats négatifs à $100 \%$. Il estimait que cette expérience avait été très positive.

A mon premier séminaire, j'ai pensé à cette expérience et j'ai dit à mes étudiants : "Cherchez à la bibliothèque tous les livres qui ont un rapport avec votre intérêt musical particulier et lisez-les de façon critique. Chaque fois que l'auteur écrit «sans aucun doute ", "c'est incontestable », «ce n'est pas la peine d'expliquer davantage », « ceci est un a priori » ou d'autres suppositions de ce genre, qui ne sont basées sur aucune documentation, notez-le et ajoutez un point d'interrogation. Cela signifie probablement que l'auteur n'y connaît pas grand'chose ou que le sujet est peu connu. A la fin de votre lecture vous aurez établi ce que j'appelle une " prémisse négative ", c'està-dire une thèse postulant qu'il y a peu ou presque pas de connaissances concernant un sujet donné. Une telle prémisse peut former une base positive et constructive pour une recherche qui réussira à répondre à des questions qui ne sont pas encore formulées. Voici une recherche originale!»

Cette attitude critique qui consistait à poser des questions, est devenue une tradition dans notre « Séminaire principal » et a lancé d'innombrables projets de recherche. Mon avertissement était : "Comment voulez-vous trouver une réponse si vous ne posez pas une question? » Ainsi, au début de notre programme, nous avons consacré beaucoup de temps à formuler des questions.

La base du programme à UCLA était qu'il fallait absolument apprendre en «mettant la main à la pâte ». Apprenez, aussi modestement que ce soit, à chanter et/ou à jouer la musique qui vous intéresse. Bien plus tard, j'ai entendu énoncer, sous la direction de Charles Seeger, que la parole et la musique sont vraiment deux modes du discours, mais deux modes différents! Nos groupes de musique et de danse (ces deux sujets sont souvent inséparables) sont devenues à juste titre célèbres. Elles maintenaient un très 
haut niveau par respect pour les traditions qu'elles représentaient. N'étant pas euxmêmes les héritiers de ces traditions, nos étudiants interprètes, dirigés généralement par un bon musicien-danseur de la culture qu'ils étudiaient, étaient conscients de la nécessité d'atteindre un excellent niveau d'interprétation.

Je n'ai jamais dénombré les différentes traditions qui étaient inscrites au programme de la pratique musicale mais je pense qu'il y en avait de trente-cinq à quarante. Je me suis efforcé de mettre sur pied un groupe d'étude interprétant l'art d'une nouvelle culture, chaque fois qu'il y avait un étudiant sérieusement engagé dans l'étude de ce sujet. L'étudiant en question devenait alors l'étincelle qui inspirait les autres et donnait à l'entreprise une énergie et une viabilité contagieuses. On m'a dit que nous avons eu à un certain moment de cinq à six cents étudiants, provenant de différentes disciplines du campus, qui formaient des ensembles musicaux.

Ainsi, des groupes spécifiques d'interprétation musicale se développaient autour des intérêts des enseignants et des étudiants. Quels étaient les principaux cours d'ethnomusicologie que tous les étudiants devaient suivre ? Qu'en était-il de la formation en musique occidentale.

Plusieurs choses étaient implicites dans l'élaboration d'un programme de formation dans un domaine comme l'ethnomusicologie. Dans la plupart des cas, il n'existait pas de musiques écrites ou, s'il y en avait, c'étaient des partitions sommaires ou des esquisses de la musique qui était effectivement jouée. Par conséquent, il était clair que nous devions mettre l'accent sur la documentation professionnelle: enregistrement sonore, photographie, film documentaire, formes originales d'annotations musicales, quand il le fallait, et sur un très bon style d'écriture en anglais. Ici, le mot clef est " professionnel ». Que de fois n'a-t-on pas allégué des excuses inacceptables pour des enregistrements et des photos médiocres ou franchement mauvais (au début le riche potentiel des films documentaires était encore inexploré).

En Occident, les études musicales se sont développées grâce aux compositeurs, interprètes, théoriciens, professeurs et enfin "musicologues». Bien qu'il aie fallu dépasser un préjugé eurocentriste, il était tout aussi essentiel que les ethnomusicologues connaissent les nombreuses traditions musicales occidentales pour pouvoir communiquer avec le monde spécialisé de la musique. Les développements de la musique occidentale ont certainement été différents de ceux des autres parties du monde; mais l'étude scientifique de ces développements a été propre au monde occidental. C'est pourquoi j'estimais que la connaissance des traditions musicales de l'Occident était une condition sine qua non. Une connaissance approfondie des traditions de la musique occidentale, une compétence vraiment professionnelle dans les diverses techniques de la documentation de base, une connaissance pratique, « en mettant la main à la pâte ", de plusieurs cultures musicales non-occidentales et la spécialisation dans l'une d'elles. C'est vrai que le verre est bien plein... mais de quel riche breuvage !

Pourriez-vous évoquer l'atmosphère des «fameux » Séminaires principaux du mercredi, en ethnomusicologie.

Vos questions suscitent des réponses trop longues! Je vais essayer d'être bref. il y avait généralement de quatre à sept, jamais moins de trois, professeurs qui représentaient une grande variété de disciplines : ethnomusicologie, musicologie, anthropologie, génie civil, physique, géophysique, histoire de l'art, linguistique. Cela offrait une base riche, faite de points de vue différents, permettant l'examen de n'importe quelle question fondamentale de la recherche. Le résultat était que nous avions des séminaires très 
ouverts ; le statut d'enseignant ou d'étudiant importait peu. Nous discutions librement entre nous, parfois même nous nous disputions, mais toujours de manière constructive, de sorte que les questions trouvaient toujours une réponse satisfaisant plusieurs points de vue différents. D'après Charles Seeger, je jouais le rôle d'un orchestrateur qui devait veiller à ce que les participants ne s'éloignent pas des mêmes aspects d'un sujet donné. C'est ainsi que j'ai créé la fameuse ligne « G-S » : tout sujet contient en lui une ligne continue de sous-thèmes qui peuvent être arrangés du plus Général au plus Spécifique. Pour qu'une discussion soit fiable, il faut maintenir les gens à peu près sur le même point de la même ligne G-S.

Rétrospectivement, je crois qu'un des traits caractéristiques du « Séminaire principal du mercredi " était l'absence totale de séparation entre étudiants et enseignants. Lorsqu'ils s'adressaient aux professeurs et parfois même contredisaient leurs affirmations, les étudiants expérimentés étaient bien entendu plus audacieux que les nouveaux venus au séminaire. Mais après une année de fréquentation, ces derniers étaient prêts à se livrer à un combat intellectuel avec n'importe quel professeur.

Je pense qu'il y avait là une cause sous-jacente qui a tendance à être absente des études du troisième cycle en musicologie. Nous avons très vite compris que nos "étudiants » étaient souvent des autorités mondiales en la matière pour une ou plusieurs traditions musicales non-occidentales. Leur connaissance était unique dans le concert de nos compétences. Nous autres, nous étions véritablement leurs élèves. Klaus Wachsmann a un jour fait allusion à cette époque en l'appelant l'«âge d'or de l'ethnomusicologie ». J'ai l'impression que cette conscience que nous avions tous d'être des apprentis formait la clef de voûte de cet âge d'or où chacun apprenait auprès des autres.

Comment était-il possible de réunir à UCLA autant de grands spécialistes et de maîtres des traditions musicales non-occidentales.

Je suppose que vous ne voulez pas seulement parler de notre faculté où nous avons été jusqu'à neuf enseignants, mais aussi des professeurs de musique et de danse issus de différentes cultures et d'un très grand nombre d'étudiants internationaux qui venaient souvent avec leur immense et remarquable connaissance du sujet, et auxquels je faisais tout à l'heure allusion. Je crois que notre programme les attirait parce que nous avions justement la réputation d'essayer d'apprendre les uns des autres. A l'assemblée constitutive de la Société américaine d'ethnomusicologie (SEM), j'ai signalé que nous ne serions jamais assez d'ethnomusicologues pour faire le travail qui devait être fait. Par conséquent, nous ne devions pas commettre l'erreur de nombreux autres humanistes, artistes et savants, en nous tirant dans le dos, en étant trop critiques et fondamentalement négatifs. Nous devrions plutôt coopérer et travailler ensemble. Je crois sincèrement que c'était cela l'esprit prédominant de ce que Wachsmann appelait l'« âge d'or » à UCLA.

Que pensez-vous du rôle de l'enseignement dans votre carrière?

Je vous répondrai brièvement en vous disant qu'à mon avis un bon enseignement exige du maître qu'il donne lui-même l'exemple. J'étais persuadé que je devais moi-même apprendre à jouer la musique et même à danser, apprendre à mener des recherches rigoureuses et approfondies, apprendre à poser des questions avec clarté, à écrire simplement et clairement, à interroger, mais sans préjugé, toutes les autorités y compris les détenteurs de la tradition. En bref, je pourrais faire deux remarques : $1^{\circ}$. Un bon professeur doit donner les exemples que l'on attend de l'étudiant ; il doit pouvoir 
anticiper les problèmes auxquels l'étudiant va s'exposer pendant la durée de son apprentissage. $2^{\circ}$. Il doit être réceptif aux leçons qu'il peut recevoir de ses étudiants. Je crois honnêtement que j'ai été un étudiant pendant toute ma vie académique. A mon avis, dès que vous cessez de réaliser à quel point il est important d'apprendre des autres, vous arrêtez d'être un bon professeur.

Quand, comment et pourquoi est apparu, pour la première fois, le concept de la bimusicalité qui est lié à votre nom et qui fait aujourd'hui partie intégrante des études ethnomusicologiques.

Le concept a été promulgué dans notre programme à UCLA et il est devenu largement connu (au début il était parfois controversé). Il s'agissait d'apprendre à jouer la musique et à exécuter la danse de son sujet de recherche parce que cela constituait la source d'information la plus fiable. Dans les années soixante, j'ai reçu un coup de fil de David McAllister qui me demandait si je voulais bien faire une intervention au congrès du SEM, dans l'Indiana, au sujet de la « bi-musicalité » (à l'origine le terme est de lui). Je lui ai répondu : «Je suis d'accord, David, et j'accepte ton titre. Il n'est pas mauvais. Mais je risque de le contester à la fin de mon exposé ». Et je l'ai fait. Bien que le terme soit largement répandu aujourd'hui (une thèse de doctorat a même été écrite sur ce sujet à l'Université de Columbia, il y a quelques années), je pense que très peu de gens ont lu l'exposé original ou bien qu'ils ne se souviennent pas de ses conclusions. Le concept est clair : vous pouvez appliquer la musicalité à n'importe quelle musique, «bi » est donc un préfixe inadéquat. Cela pourrait être «tri » ou « quadri » ou quelque chose d'autre. J'insistais simplement sur le fait que nous parlions réellement de "musicalité", appliquée à autant de traditions qu'on avait le temps d'étudier. Aujourd'hui, le concept est bien sûr accepté partout dans le monde, par exemple par la nouvelle société internationale "Teaching World Music ». Nous avons démontré à UCLA qu'une telle pratique constituait la base de toute recherche fiable en ethnomusicologie.

Aujourd'hui le gamelan est très répandu en Occident. Vous avez été le premier, ou un des premiers, à avoir fondé un gamelan hors de I'Indonésie. Quel souvenir gardez-vous des débuts de cette expérience?

Lorsque j'étais l'élève de Jaap Kunst, en Hollande, j'ai reçu un jour un coup de fil de l'attaché culturel de l'ambassade américaine me disant : « Il y a, dans mon bureau, une dame hollandaise qui voudrait rencontrer un riche américain." J'ai ri en me demandant pourquoi il m'avait appelé, j'étais un étudiant très pauvre et je vivais avec une bourse Fulbright. Il a continué : « Elle est née à Java et elle est revenue en Hollande peu avant la seconde guerre mondiale. Elle veut vendre ses bijoux et d'autres objets, dont un petit gamelan javanais. Je lui ai parlé de vous. »

Quelques jours plus tard, je suis allé à La Haye, j’ai acheté le gamelan et je me suis mis à un sérieux régime à base de macaronis et de fromage. La dame m'a fait sentir la responsabilité que j'allais désormais assumer en devenant le nouveau propriétaire du gamelan, en organisant une cérémonie de deux heures avec de l'encens, des prières javanaises et en m'offrant un cadeau de sept kriss pusaka (pouvoir magique sacré). Elle m'a dit que j'allais faire des rêves à propos du gamelan et que s'il m'arrivait malheur, il faudrait que je me débarrasse de l'instrument. Elle avait grandi à Java Central, avait étudié la danse depuis sa petite enfance à la cour royale (kraton), et avait décidé de se procurer un gamelan et de faire une tournée de danse en Europe, accompagnée de sept musiciens. Mais la guerre avait mis fin à ses projets; le conservateur du musée de La Haye avait caché le gamelan pour éviter que les Nazis ne le fassent fondre pour leurs 
besoins militaires. Maintenant, nous étions en 1954, elle savait que le moment était venu de s'en séparer.

J'ai ramené le gamelan en Californie, je l'ai baptisé Udan Mas (Pluie d'or) et j'ai formé un groupe de musiciens : un an plus tard, nous jouions devant le président Soekarno et ses soixante ministres en visite officielle aux Etats-Unis. Il est bon de savoir que c'est une hollandaise, née à Java, qui est à l'origine de la pratique musicale en ethnomusicologie, en Amérique et dans le monde entier. Ça valait la peine de se mettre au régime macaronis et fromage!

Que pensez-vous des récents développements des groupes de gamelan en Occident ? On trouve non seulement des groupes qui jouent des pièces traditionnelles, mais aussi d'autres qui combinent la tradition avec de nouvelles compositions, sous l'influence de la nouvelle musique créée à Java et à Bali.

Là on pourrait commencer un livre, Giovanni! Le développement rapide de l'intérêt pour le jeu du gamelan est remarquable, il existe plus de deux cents groupes en activité aux Etats-Unis. Harold Powers a noté, il y a quelques années, que le gamelan facilitait la participation de personnes ayant différents niveaux de compétence, il était par conséquent un excellent moyen pour introduire dans l'enseignement une musique autre que celle de la tradition artistique européenne. J'avoue que certains développements du gamelan m'inquiètent; mais, d'un autre côté, les motivations des individus et des groupes sont très variables. Les expériences avec le gamelan sont courantes dans le monde, y compris à Java et à Bali ; à mon avis, un grand nombre d'entre elles ne sont pas fondées esthétiquement. Je me console avec l'idée qu'en Indonésie, et nulle part ailleurs, la tradition se trouve entre les mains de ses créateurs; les développements sont souvent malencontreux, spécialement ceux qui découlent de contacts superficiels avec l'Occident. Peut-être pourrait-on excuser certaines de ces productions à cause de leur origine autochtone. Mais les développements internationaux dans le sens inverse sont aussi répandus. Il me semble qu'ils ne sont pas facilement justifiables.

Vous avez mené l'essentiel de votre travail de terrain en Indonésie (Java et Bali) mais aussi en Afrique et à Hawaii. Pourriez-vous nous parler de vos expériences de terrain.

En considérant ma longue carrière passée, je crois que les souvenirs qui restent les mieux ancrés dans ma mémoire sont certaines expériences d'ordre personnel que j'ai eues sur le terrain, mais que je ne publierai probablement jamais à cause de leur caractère trop personnel et intime. Je pense par exemple au jour où Hazel et moi sommes devenus chacun membres de la tribu des Fanti, au Ghana, lors d'une cérémonie commémorative en l'honneur du fils d'un souverain. Je ne vais pas tout raconter ici, mais je n'oublierai jamais la petite reine-mère des Fanti qui mesurait $1,50 \mathrm{~m}$ et qui avait des yeux merveilleusement expressifs. Pendant toute la durée de la cérémonie, notre seule façon de communiquer était de nous regarder dans les yeux. C'est un événement que je n'oublierai jamais.

A Hawaii, j'ai bien connu plusieurs des fameuses Tantes (la société hawaïenne est matriarcale). L'une d'elles était une mystique, une autre était une intellectuelle, dépositaire d'anciennes traditions, une autre encore était une musicienne très spéciale, une chanteuse qui avait beaucoup de mana. J'ai appris beaucoup de choses de ces femmes, certaines étaient sacrées aux yeux des quelques Hawaïens qui en étaient conscients, d'autres étaient très personnelles, je ne les publierai jamais, mais jamais non plus je ne les oublierai. 
Je crois que ces expériences exemplaires révèlent les aspects les plus vivants du processus d'apprentissage; elles sont des lumières, apportées par la recherche, qui informent le chercheur mieux que n'importe quoi d'autre. Elles donnent un sens profond à la musique, à la danse, à la littérature, à la poésie, au rituel et à la religion. Elles constituent les valeurs intrinsèques des témoignages qui m'ont été révélés pendant mon travail sur le terrain, à Bali, à Java, en Afrique et à Hawaii. Même si un jour l'idée d'écrire un livre à ce sujet devenait une réalité, je ne publierais jamais ces souvenirs du terrain, que je garde comme un trésor dans ma mémoire.

Fig. 2 : Discussion avec Mampoma, Honolulu, 1981, congrès de l'IFMC.

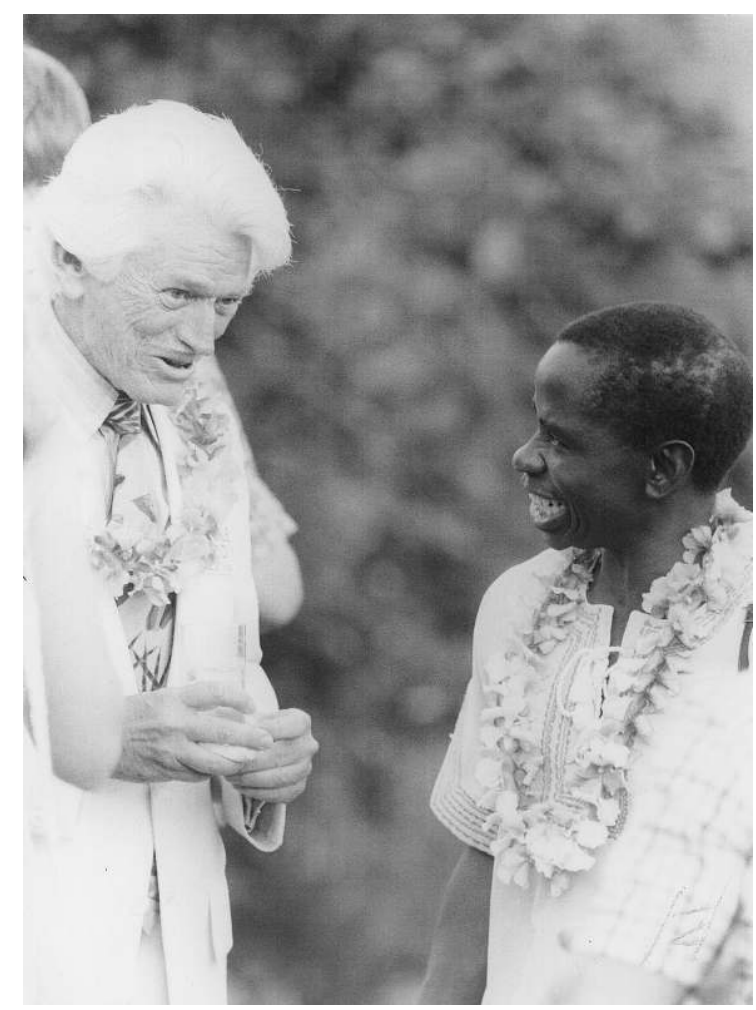

Photo : Folklore Film, Drottninggat, 85, 2 tr. 08-20 75 04, S-III 60, Stockholm, Suède.

Y a-t-il, à Bali et à Java, des maîtres de musique dont vous vous souveniez avec affection et respect?

Oui vos questions vont bientôt éveiller le désir d'écrire un livre ! Je dirai seulement que j'ai connu beaucoup de maîtres dont j'estime profondément l'amitié et la générosité. $\mathrm{Si}$ je n'en cite ici que quelques uns, c'est par souci de concision. Le regretté Pak Pantjopangrawit était un grand joueur de rebab et de gender, spécialement de rebab. Ses improvisations étaient considérées par tous ceux qui ont eu le privilège de l'entendre comme un sommet dans l'art de l'improvisation imaginative javanaise. Je me souviens de lui avec amour, comme de mon maître et ami intime. Le maître de cérémonies au palais du Sultan à Jogya et directeur du gamelan de la cour royale, K.R.T. Madukusuma, auquel j'ai dédié le deuxième volume Legacy of the Roaring Sea (1984) de ma trilogie, The Evolution of Javanese Gamelan, a été le fidèle compagnon de beaucoup de mes recherches sur le terrain, en 1957-58, et une constante source d'inspiration comme gardien du flambeau de la tradition javanaise. Je mentionnerai encore une troisième personne, Pak Tjokro, qui était incontestablement le maître javanais le plus dévoué que j’ai connu ; il a 
longtemps enseigné à l'Institut des Sciences Humaines de Californie, avant de se retirer à Java où il a maintenant plus de 80 ans. Je lui ai dédié mon troisième volume, Paragon of the Roaring Sea (1988). Sa patience, son enseignement et son amitié constituent les biens les plus précieux de mes études de musique javanaise, depuis notre première rencontre à la RRI (Radio de la République Indonésienne), en janvier 1957. Je me limite à trois noms, je pourrais en citer dix fois plus!

Je me souviens d'une phrase de Kunst que vous m'avez une fois rapportée et qui reste depuis ancrée dans mon esprit. Kunst disait qu'en apprenant la langue de la culture dont on étudiait la musique il fallait en premier apprendre à dire : «êtes-vous fatigué ? » Quelle est pour vous l'importance des relations personnelles que l'on établit avec les musiciens pendant la recherche sur le terrain? Avez-vous des suggestions à faire aux étudiants qui commencent leur travail de terrain?

Cette phrase simple, recommandée par Kunst, contient implicitement la réponse à votre question. Je voudrais rappeler à tous ceux qui envisagent de faire des recherches sur le terrain que notre succès relatif dépend en définitive de l'amitié, de la patience, de l'intérêt, de la compréhension, de l'estime et de la chaleur que nous accordent les personnes avec lesquelles nous avons le privilège de former des liens personnels étroits. On a dit que j'étais farouchement opposé à l'emploi du mot «informateur » pour désigner ces personnes, parce que, littéralement, la signification de ce terme se limite à une méthodologie pratique en linguistique. La personne avec laquelle l'ethnomusicologue travaille, crée des liens d'amitié et parfois même d'amour, est avant tout un être humain, avec toute la force et la faiblesse que ce mot suggère, un musicien, un danseur, un conteur, une personnalité religieuse, quelqu'un doté spécialement de mana (pouvoir magique et force morale). Un informateur? Oui, les informateurs aussi se fatiguent, parce qu'ils sont avant tout des être humains. Si l'on conserve précieusement l'équation humaine comme hypothèse de départ du travail sur le terrain, «êtes-vous fatigué ?» révèle ce début d'intérêt qui présage une relation personnelle durable.

Les questions qui relèvent de l'ethnomusicologie appliquée sont, elles aussi, en rapport avec la recherche sur le terrain. L'ethnomusicologue est souvent confronté, sur le terrain, à des problèmes spécifiques qu'il est appelé à résoudre. Dans votre article «Effects of Medieval Technology », vous racontez votre tentative de faire revivre les grands gongs à Java. D'après votre expérience de terrain, que devront être, selon vous, le rôle et l'attitude des ethnomusicologues dans ce genre de situations?

Votre question découle logiquement de la question précédente. Si en tant que chercheur, vous êtes mû par le désir sincère de vous faire des amis sur le terrain et non pas seulement d'identifier des "informateurs", vous vous sentirez alors prêt à aider vos nouveaux amis du mieux que vous pourrez. L'histoire de faire revivre le grand gong avait commencé bien avant que je n'arrive à Java. La triste déclaration de Kunst, qui constatait dans une note additionnelle à son livre Music in Java ( $2^{\mathrm{e}}$ éd.) que l'art de fabriquer le grand gong était perdu, m'avait frappé comme une perte personnelle. Je me sentais préparé, par la lecture d'une étude de la fin du XIXe siècle, et déterminé, malgré les réactions toujours négatives suscitées par mes premières tentatives à Java, à le voir restauré. Mon sentiment était si fort que j'osais m'opposer à mes amis javanais qui prétendaient que cela ne pourrait pas se faire. Je me sentais engagé et très déterminé. Naturellement, nous avons tous été ravis lorsque ces deux années de persévérance ont porté leur fruit. 
Mais, mis à part le domaine de la musique et des arts qui lui sont liés, l'ethnomusicologue peut aussi aider de diverses manières ses amis sur le terrain. Il m'est arrivé une histoire dont je n'ai jamais parlé dans mes publications, chez les Sundanais de l'ouest de Java qui m'avaient invité à visiter une station expérimentale d'agricultutre non loin de Bandung. Ils avaient des difficultés à faire pousser du raisin qui devenait trop acide ; ils m'ont demandé de rester quelques jours chez eux pour leur donner des conseils à ce sujet. Apparemment quelqu'un avait dû remarquer, à Jakarta, que j'avais publié quatre articles dans le domaine de l'agriculture et espérait que je pourrais les aider. Je pense que je l'ai fait, en leur expliquant simplement, après avoir goûté la grappe de raisin la plus grande et la plus acide de ma vie, qu'il pleuvait beaucoup trop dans la région de Bandung. Je leur ai conseillé d'abandonner la vigne et d'essayer une autre culture. Il l'ont fait !

Dans le Harvard Dictionary of Music, vous avez donné une définition de l'ethnomusicologie qui a été, et qui reste toujours, une référence: "L'ethnomusicologie est une approche à l'étude de toute musique, non seulement pour elle-même, mais aussi en relation avec son contexte culturel ». Est-ce que vous êtes toujours d'accord avec cette définition? Sinon, quel changement souhaiteriez-vous y apporter?

Je me souviens de la définition. Elle a été approuvée par l'examen minutieux de Charles Seeger et elle reste toujours valable. N'importe quelle définition, réduite à une seule phrase, omet nécessairement beaucoup de choses.

Comme je dispose ici de plus de place qu'une seule phrase, j'aimerais souligner à quel point il est essentiel de tenir compte des systèmes de valeurs. Les valeurs établies par la société donnent un sens à la musique et aux arts apparentés et sont en même temps profondément gravées dans l'identité des individus d'âges et de comportements divers. Bien entendu, le terme de « valeurs » est inclus dans l'expression « contexte culturel ». Mais trop souvent (je pense ici aux avertissements donnés par Seeger), le chercheur limite ses efforts à l'accumulation des faits, en oubliant, ou en ignorant intentionnellement, le truisme qui veut que les faits soient inséparables des valeurs qu'ils représentent. Il faut apprendre très attentivement auprès des dépositaires de la tradition tout ce que l'on peut connaître sur les valeurs que la tradition transmet aux personnes et aux groupes vivant au sein d'une société. Il ne faut pas négliger les valeurs parce qu'on est impatient d'accumuler les faits. Un « fait » ne signifie pas grand-chose si l'on ne reconnaît pas consciemment la valeur qui lui est corrélative.

On devrait étendre la définition de façon à ce qu'elle englobe une prise de conscience de l'existence d'une sagesse qui est, en tout cas dans sa manière de s'exprimer, propre à chaque société. J'ai trouvé, à Java, à Bali, en Afrique et à Hawaii, des mots et des phrases qui n'appartiennent qu'à leurs langues respectives. Ils symbolisent des concepts qui non seulement ne peuvent pas être traduits dans un autre idiome, mais n'existent peutêtre même pas dans des termes approximatifs dans une autre culture.

Pour expliquer un dernier point concernant les définitions en une seule phrase, je dirai quelques mots sur un mode de pensée qui a guidé mes recherches pendant ces vingtcinq dernières années. J'ai repoussé le moment de parler du mode de pensée jusqu'à la publication de The Ethnomusicologist (1971) où j'ai introduit le terme d' «indicibles » ( untalkables). C'est seulement en 1989 que je me suis aventuré à publier « Music from Galileo to Einstein. A Quantum Leap ». Ensuite, dans une série d'exposés sur la théorie quantique de la musique (1990 [Berlin], 1991 [Amsterdam], 1992 [Trieste], 1993 [Calella]), j'ai tâché d'expliquer un peu plus ce mode de pensée dans la recherche. Je 
l'appelle un "mode de pensée " parce qu'il attire l'attention sur les innombrables facettes de la musique, des arts qui lui sont apparentés et des sociétés qui les ont engendrés et qui ont longtemps été ignorés, par manque de sensibilité ou à cause d'une conviction erronée qui ne leur accordait aucune importance. Je crois qu'il faudrait éventuellement résumer ma définition en une seule phrase, de manière à attirer l'attention sur le fait que la Théorie quantique de la Musique (TQM), comme l'ont surnommée «les Six», est un mode de pensée fondamental pour tout ethnomusicologue sérieux.

On vous a souvent opposé à Alan Merriam pour votre différence d'approche dans vos recherches ethnomusicologiques. Quelles étaient les causes de vos différends?

Je voudrais dire pour commencer que les soi-disant désaccords entre Merriam et moi étaient souvent exagérés et utilisés à mauvais escient par des personnes qui souhaitaient brouiller les ethnomusicologues ayant une formation anthropologique et ceux qui étaient au départ des musicologues. Je pense que nous avons prouvé notre respect mutuel pour un savoir acquis par des approches différentes lorsque Alan m'a invité à enseigner pendant une année, en 1977-78, à l'université d'Indiana. La différence fondamentale entre nos approches résidait dans le fait qu'Alan considérait «l'homme " comme son sujet et la musique comme un facteur essentiel pouvant fournir des informations uniques sur ce sujet. Tandis que, pour moi, la musique constituait le sujet et je considérais les systèmes de valeurs de l'homme comme étant essentiels à la compréhension complète de ce sujet.

La principale différence d'approche était ma foi inébranlable dans le fait que le chercheur devait devenir l'interprète de la musique et de la danse qu'il étudiait et qu'une approche pratique, " en mettant la main à la pâte ", était essentielle pour que la recherche soit fiable. Alan estimait qu'il n'était pas vraiment utile pour le chercheur de s'investir dans l'interprétation de la musique et de la danse étudiées. Il a quand même reconnu, dans un débat organisé par le directeur de l'Institut de folklore de l'Université d'Indiana en 1978, que cela pouvait avoir une valeur « limitée ».

La deuxième différence d'approche concernait notre façon d'envisager les publications du passé sur un sujet donné. Je les ai toujours regardées avec un respect critique, reconnaissant qu'elles constituaient un aspect indispensable de la recherche. Mais les recherches qui m'intéressaient ont été pour la plupart (il y a bien sûr des exceptions) tournées vers l'avenir. Je m'intéressais aux écrits scientifiques du passé, mais j'étais cependant convaincu qu'ils dataient d'une époque antérieure et dépassée en ce qui concerne l'attitude scientifique (la fin du colonialisme) et philosophique (nous pouvons apprendre de toutes les sociétés).

Alan respectait beaucoup la science du passé et il avait raison. Mais en lisant par exemple The Anthropology of Music, je trouve qu'il n'était pas assez attentif aux temps qui changent et que le lecteur risque d'être ainsi tenté de charrier ces théories et méthodes au présent et à l'avenir. Dans ce livre, il passe longtemps en revue la théorie anthropologique des cinquante dernières années, ce qui a bien entendu une valeur indiscutable. Mon approche, par exemple dans The Ethnomusicologist, concernait au contraire la théorie actuelle et ses implications dans la recherche future. J'ai souvent pensé que si nous avions collaboré, Alan et moi, dans la recherche d'un même sujet, nous aurions produit à nous deux quelque chose de vraiment exceptionnel. 
Dans les années 1960, vous avez collaboré avec Seeger au projet d'un Mélograph. Depuis, vous vous êtes constamment intéressé aux aspects du son dans la musique, en étant particulièrement attentif aux éléments du son qui dépassent les paramètres conventionnels.

Nous avons élaboré ensemble, Charles Seeger et moi, le plan du Modèle B du Mélograph de Seeger. Lorsque nous l'avons fini, je lui ai dit: "Charles, nous avons omis l'aspect le plus important du son musical, le spectre partiel, la vraie "qualité du son » ». Par la suite, nous avons cherché pendant plusieurs années à trouver des fonds pour construire le Modèle $C$, à savoir 5000 dollars pour l'étude de faisabilité et 125000 dollars pour la fabrication. C'était beaucoup d'argent au début des années soixante, et ça l'est toujours encore! Ce Mélograph a permis de nombreuses thèses et mémoires ainsi que des projets de recherche indépendants.

Grâce aux leçons de cette période, nous avons pu améliorer notre équipement de laboratoire et nous l'avons développé à l'Université du Maryland, Comté de Baltimore (UMBC), pendant une douzaine d'années, à partir de 1981. Il y a dix ou douze ans, vous vous souvenez, l'Université John Hopkins nous a envoyé un médecin, le docteur James Bosma, spécialiste des "sons des cris de bébé ", qui a pu sauver des vies grâce à des sonogrammes plutôt limités. John Hopkins avait recommandé notre laboratoire comme étant le plus développé de cette partie du pays. Plusieurs d'entre vous ont travaillé avec succès avec le docteur Bosma, et les recherches qu'il mène à l'heure actuelle avec Frank Vice ont obtenu des résultats étonnants, qui dépassent de loin mes compétences.

Au début de notre entretien, vous avez dit que ce qui vous avait le plus fasciné était le «son » de la musique javanaise. Depuis ce moment, la recherche sur le son a suscité chez vous un vif intérêt. D'après vous, quelles sont les questions les plus intéressantes que vous vous êtes posées au sujet du son javanais et balinais pendant votre carrière, quelles en sont les réponses et quelles sont les questions qui restent encore sans réponse?

Les bons musiciens de Java et de Bali reconnaissent immédiatement le son de tel ou tel gamelan célèbre, qu'ils distinguent aisément de tous les autres. Comment font-ils? Après tout, nous pourrions supposer que le son du bronze frappé (il existe seulement trois différentes qualités reconnues de bronze utilisées pour le gamelan) est le même d'un gamelan à l'autre. Ce n'est pas vrai ! L'alliage particulier de l'étain, du cuivre et d'infimes quantités d'or ou d'argent, l'âge des lames et des gongs, la quantité de «travail » (jeu) qu'il a subie, le processus d'accordage, l'échelle d'intervalles qu'il représente, la proportion d'étirement et de compression des octaves, l'ombak ou battement (dans les ensembles balinais et dans les vieux ensembles de Java) entre lames de même hauteur, l'angkep-angkepan (voir mon récent article sur le sujet) : ce sont là quelques unes des variables qui me viennent à l'esprit. Par conséquent, lorsque j'ai dit que ce qui me fascinait était le son du gamelan, je me réferais à la remarquable individualité $\mathrm{du}$ son de chaque gamelan. Au début, n'ayant connu que la musique occidentale, je n'étais pas conscient de toutes ces infimes différences, mais je les ai entendues! Et je les entends de mieux en mieux !

Il faut ajouter à ces caractères l'individualité des ensembles humains jouant du gamelan. Leur façon particulière de produire le son du bronze frappé est une autre variable. Il y a longtemps, nous avions à UCLA, dans mon petit gamelan javanais Udan Mas, un jeune étudiant diplômé en droit international qui jouait du kethuk. Comme vous savez, cet instrument est un petit gong avec une bosse centrale, qui rend un son étouffé lorsqu'on le frappe avec le tabuh. On pourrait présumer hâtivement que son registre sonore est nul. Il n'en est rien! Pendant six mois, Niel a fait des expériences pour 
découvrir avec précision l'endroit de la bosse qui devait être frappé, la technique de frappe, la position dans laquelle l'instrument doit être suspendu sur des cordes croisées dans son support, pour produire ce qu'il estimait être le meilleur son. Bon, si un tel instrument a potentiellement autant de variables, imaginez combien de variables existent dans des instruments comme les trois tailles du bonang ou les deux tailles du gender ou du slenthem, dans des tambours kendang, dans le rebab (vièle) ou le gong agung (le plus grand gong)! Le son du gamelan? Quelle riche expérience, si l'on sait vraiment en comprendre les potentialités!

Vous avez mentionné tout à l'heure le terme d'« indicibles » (untalkables), que vous avez déjà employé dans The Ethnomusicologist (1971). Qu'est-ce que cela signifie?

Je crois qu'une bonne partie de ce que j'ai énuméré dans ma dernière réponse pourrait s'intituler: les « indicibles ». Mettons nous d'accord: le terme, tel que je l'emploie, n'a rien d'absolu ou d'exclusif. Par exemple un certain nombre d'indicibles que j'avais essayé de décrire il y a quelques années ont été rendus "visibles ", grâce à une série d'améliorations apportées au Mélograph (Model C, Poly « D » et les modèles ultérieurs). En d'autres termes, les "indicibles" sont tous ces aspects, non seulement du son musical mais aussi de la pratique musicale, qui ont de profondes répercussions sur la musique. La plupart d'entre eux n'ont pas été reconnus par la musicologie conventionnelle comme étant particulièrement significatifs, si tant est qu'ils aient jamais été reconnus! En employant ce terme, j'essaie de diriger l'attention du chercheur vers des facteurs subtils qui déterminent la musique, en tant que son et en tant que pratique. J'espère que nous en serons tous un jour conscients, si nous réussissons une fois à trouver des moyens pour les montrer et les décrire. En un sens, reconnaître les indicibles c'est accepter le vieil adage chinois qui veut que la musique puisse être représentée par un son unique.

Récemment vous avez proposé, dans certains de vos articles, la recherche d'une «Théorie quantique de la musique ». Qu'entendez-vous par là?

Mes deux réponses précédentes répondent implicitement à cette question. Chez moi, l'emploi du terme de «théorie quantique » découle du même esprit que celui qui devait motiver, je crois, les scientifiques des années 1920 ; c'est-à-dire qu'ils n'étaient d'accord que sur la seule nécessité de poser le principe d'une théorie quantique. Ils cherchaient tous les moyens d'en parler mais ils n'étaient pas sûrs des termes qu'ils devaient employer. Par moment il semble même qu'ils étaient bloqués dans leurs réflexions, mais ils étaient tous d'accord sur un point : il fallait continuer à réfléchir !

Je crois que nous sommes un peu plus avancés que cela dans la théorie quantique de la musique. En répondant à vos deux questions précédentes, j'ai identifié plusieurs aspects de la musique dont on ne tient pas encore compte lorsqu'on étudie une tradition musicale donnée. Pour qu'elle soit pertinente, une théorie quantique de la musique doit englober tous ces facteurs et très probablement beaucoup d'autres dont nous ne sommes pas encore conscients. C'est pourquoi je suis persuadé que nous sommes en train de trouver notre chemin. Nous ne pouvons pas, comme on me l'a demandé à Trieste, il y a deux ans, écrire notre formule de la TQM sur un tableau noir. Pourtant il se peut que même cette éventualité se réalise un jour, lorsque nous serons sûrs d'avoir identifié tout ce qui est essentiel à une étude viable de la musique. Je suis optimiste et je crois que nous réussirons à publier quelque chose d'assez avancé pour provoquer au moins la réaction de ceux qui, plus que nous encore, sont perplexes au sujet de ce que nous essayons de faire. 
Vous vous êtes intéressé depuis très longtemps à l'improvisation.

Oui et je m'y intéresse toujours. Là encore nous avons affaire à un champ d'étude qui implique très certainement la présence de nombreux indicibles. Mon expérience du jazz, il y a bien des années, était excellente mais s'est avérée bien insuffisante lorsque j'ai commencé à observer les phénomènes liés à l'art de l'« improvisation de groupe » du gamelan javanais. L'improvisation est l'âme même du gamelan javanais mais, contrairement au jazz, elle ne concerne pas un soliste soutenu par un groupe. A Java, $70 \%$ à $80 \%$ des joueurs de gamelan improvisent simultanément. Comment réussissent-ils à ne pas provoquer d'anarchie musicale alors qu'ils parlent (musicalement) tous en même temps ? J'ai commencé mes recherches sur le terrain à Java, en 1957-1958, en recueillant des enregistrements spéciaux d'improvisations faites par des professionnels, en étudiant personnellement les techniques vocales et instrumentales, en prenant trois ou quatre leçons de danse par semaine pendant presque deux ans. Ce n'est qu'après avoir réfléchi sur ces matériaux pendant les huit années qui ont suivi qu'une "lumière » intellectuelle s'est enfin allumée et que j'ai compris les bases de l'improvisation de groupe. Les améliorations apportées à la théorie et la publication concrète des résultats n'ont pas eu lieu avant 1988 (Livre III, Paragon of the Roaring Sea de ma trilogie The Evolution of Javanese Gamelan). Je ne crois pas pouvoir ajouter à cette publication plus que ce dont il a été question tout à l'heure. Il existe certainement, dans le processus de l'improvisation de groupe, des facteurs indicibles que l'on ne pourra jamais étayer par des documents objectifs, par exemple : 1) les effets produits par un improvisateur sur les autres musiciens pendant le concert; 2) les effets de l'environnement et de l'atmosphère (humidité relative, l'heure du jour et de la nuit, la saison etc.) ; 3) l'état mental et spirituel de chaque personne ; 4) et de l'ensemble des personnes agissant les unes sur les autres, etc., etc. On pourra naturellement objecter que ce sont là pour la plupart des impondérables. J'aimerais faire deux remarques : 1) ils sont indicibles ; 2) individuellement ou collectivement, ils affectent profondément la musique faite au cours d'une improvisation de groupe. J'ajouterai encore autre chose : je garde en moi comme un trésor une expérience extraordinaire que j'ai eue en jouant de la musique javanaise : la capacité de tenir simultanément des « conversations multimusicales » avec d'autres improvisateurs. Je ne connais aucune autre tradition musicale où cela soit le cas.

Ces derniers temps, vous participez activement aux réunions du Séminaire européen d'ethnomusicologie (SEEM). Est-ce que vous avez trouvé qu'il y avait des différences d'approche entre l'ethnomusicologie européenne et l'américaine ? Le trouvez-vous encore?

Je vous répondrai indirectement mais j'essaierai quand même d'être précis. J'ai renoncé, il y a deux ans, à participer à toutes les organisations (AMS, SEM, IMS, ICTM) sauf au SEEM, probablement pour diverses raisons : vision trop étroite (exclusivement consacrée à la tradition classique européenne), trop peu d'intérêt pour la seule musique (exposés consacrés à ce qui entoure la musique, ne considérant pas la musique comme sujet central), géographiquement trop limité, trop politique. On m’objectera rapidement qu'il y a des exceptions individuelles dans chaque association; je suis le premier à être de cet avis : il y a, Dieu soit loué, des exceptions notables. Mais j'ai finalement décidé que je perdais mon temps en participant à des associations où je ne pouvais admirer que des exceptions. Les membres du SEEM mettent toujours l'accent sur la musique. C'est le sujet auquel je donne la priorité en ethnomusicologie. 
En parlant du SEEM, je ne peux m'empêcher de penser à John Blacking. Vous étiez en très bons termes et c'est lui qui vous a invité, la première fois, à participer au SEEM.

Oui, nous étions de très bons amis, John et moi ; chacun de nous admirait les pricipes directeurs de l'autre et reconnaissait leur importance. Je pense que John était avant tout un musicien. Je crois aussi que sa formation anthropologique renforçait sa conviction selon laquelle la musique était importante en elle-même et n'entravait pas le moins du monde son évaluation de la place de la musique chez l'homme. On ne sait généralement pas que je l'ai invité, il y a quelques années, à venir nous rejoindre à l'Institut d'ethnomusicologie à UCLA. Il a accepté à ma grande joie, mais six mois plus tard (il était toujours en Afrique du Sud) des obligations personnelles l'ont empêché de se joindre à nous. Plus tard, nous avons tous deux convenu que la perte avait été réciproque.

Une dernière question : que signifie le titre $K i$ ?

Le titre $K i$ me fait penser à Pandit, titre honorifique que les Indiens donnent à leurs maîtres vénérés et à leurs hommes d'Etat. En Indonésie, le terme est réservé, semble-til, aux maîtres. J'ai entendu dire que j'étais le seul non Indonésien ayant l'honneur de recevoir ce titre. En général on le traduit par "vénérable» (par exemple Khjai (javanais) Mendung, le fameux gamelan de UCLA, signifie le « vénérable nuage noir »).

\section{BIBLIOGRAPHIE}

HOOD Mantle, 1954, The Nuclear theme as a Determinant of Pathet in Javanese Music. Groningen : J.B. Wolters.

HOOD Mantle, 1959, «The Reliability of Oral Tradition ». Journal of the American Musicological Society XII : 201-209.

HOOD Mantle, 1959, « Indonésienne (Musique) ». Encyclopédie de la Musique Fasquelle II : 547-550.

HOOD Mantle, 1960, « The Challenge of "Bi-musicality” ». Ethnomusicology IV $2:$ 55-59.

HOOD Mantle, 1961, « The Javanese Rebab ». Hinrichsen's Musical Yearbook, Proceedings of the First Congress of the Galpin Society, vol. II : 220-226.

HOOD Mantle, 1963, «The Enduring Tradition : Music and Theater in Java and Bali » in Ruth McVey, ed. Indonesia. New Haven : Yale University Press : 438-560.

HOOD Mantle, 1966, « Sléndro and Pélog Redefined ». Selected Reports in Ethnomusicology I 1 :28-47. HOOD Mantle, 1969, « Ethnomusicology » Harvard Dictionary of Music, 2nd ed. Cambridge : Harvard University Press : 298-300.

HOOD Mantle, 1970, « The Effect of Medieval Technology on Musical Style in the Orient ». Selected Reports in Ethnomusicology I $3: 147-170$.

HOOD Mantle, 1971, The Ethnomusicologist. New York : McGraw and Hill

HOOD Mantle, 1971, « Aspects of Group Improvisation in the Javanese Gamelan » in Musics of Asia. Manila : National Music Council. 
HOOD Mantle, 1974, « Improvisation in the Stratified Ensembles of Southeast Asia ». Selected Reports in Ethnomusicology II 2 : 25-33.

HOOD Mantle, 1980, Music of the Roaring Sea. The Evolution of Javanese Gamelan. Book I. Wilhelmshaven : Heinrichshofen.

HOOD Mantle, 1980, «Indonesia ». The New Grove Dictionary of Music and Musicians, 6th ed. IX : $167-173 ; 215-220$.

HOOD Mantle, 1980, « Southeast Asia ». The New Grove Dictionary of Music and Musicians, 6th ed. XVII : 762-767.

HOOD Mantle, 1982, The Ethnomusicologist. New Edition. Kent, Ohio : Kent State University Press. HOOD Mantle, 1983, « Musical Ornementation as History : the Hawaiian Steel Guitar ». Yearbook for Traditional Music $15: 141-148$.

HOOD Mantle, 1984, Legacy of the Roaring Sea. The Evolution of the Javanese Gamelan. Book II. Wilhelmshaven : Heinrichshofen.

HOOD Mantle, 1988, Paragon of the Roaring Sea. The Evolution of the Javanese Gamelan. Book III. Wilhelmshaven : Florian Noetzel Verlag.

HOOD Mantle, 1989, « Music from Galileo to Einstein-A Quantum Leap ». Progress Reports in Ethnomusicology II 3.

HOOD Mantle, 1989, «L'arte della composizione e dell'improvvisazione nella musica tradizionale giavanese » in Diego Carpitella, ed. Ethnomusicologica. Siena : Accademia Musicale Chigiana : 91-106.

HOOD Mantle, 1990, « Balinese Gamelan Semar Pegulingan. The Modal System ». Progress Reports in Ethnomusicology III 2.

HOOD Mantle, 1990, « The Quantum Theory of Music ». European Seminar in Ethnomusicology VII, International Institute for Comparative Music Studies and Documentation : 273-277.

HOOD Mantle, 1992, « Voiceprints in Omani Traditional Music » in African Musicology : Current Trends, a Festschrift for J.H.Nketia, vol II. Los Angeles : University of California Press : 191-244. HOOD Mantle, 1993a, « The Untalkables of Music ». EM annuario degli Archivi di Ethnomusicologia dell'Accademia Nazionale di S. Cecilia I : 137-142.

HOOD Mantle, 1993b, « Stratification polyphonique dans les musiques d'Asie du Sud-Est ». Cahiers de musiques traditionnelles 6 : Polyphonies : 3-10.

HOOD Mantle, 1995, « Angkep-angkepan » in Dehoux Vincent, Suzanne Fürniss, Sylvie le Bomin, Emmanuelle Olivier, Hervé Rivière, Frédéric Voisin, eds, Ndroje balendro. Musiques, terrains et disciplines : 321-336. Paris : SELAF ; Louvain : Peeters.

HARRISON Frank, HOOD Mantle et PALISCA Claude Victor, 1963, Musicology. New Jersey : Prentice-Hall

HOOD Mantle et SUSILO Hardja, 1967, Music of the Venerable Dark Cloud. Los Angeles : Institute of Ethnomusicology. UCLA.

HOOD Mantle et MACEDA José, 1972, Music. Leiden ; Köln : E.J.Brill [Handb. der Orientalistik 6] 


\section{NOTES}

1. Traduit de l'anglais par Ramèche Goharian. 